\title{
Clear aligner therapy for adult patient - A case report
}

\author{
Bharathi VS ${ }^{1}$, Dharmesh $\mathbf{H S}^{2}$, Kiran $\mathbf{H}^{3 *}$ \\ ${ }^{1,2}$ Associate Professor, ${ }^{3}$ Professor, Dept. of Orthodontics \& Dentofacial Orthopedics, Raja Rajeswari Dental College and Hospital, \\ Bangalore, Karnataka, India
}

*Corresponding Author: Kiran $\mathbf{H}^{3}$

Email: kiranortho@gmail.com

\begin{abstract}
Aligners have become the alternative treatment of choice in adult patients who do not want fixed orthodontic appliance therapy due to esthetic concerns. This case report demonstrates the effectiveness of Clear aligners used to treat an adult female patient with severe proclination and generalized spacing in the upper and lower arches, good periodontal condition and a high DMFT Index. Predicted results were achieved with the clear aligners as per the virtual setup with reduction in proclination, closure of generalized spacing and a pleasing facial profile with competent lips.
\end{abstract}

Keyword Clear Aligner, Adult patient, Periodontally compromised.

\section{Introduction}

The clear overlay orthodontic appliances was introduced in 1946, when Kesling devised the concept of using a series of thermoplastic tooth positioners to progressively move malaligned teeth. ${ }^{1}$ In the recent years, increasing number of adult patients have sought orthodontic treatment and expressed a desire for esthetic and comfortable alternatives to conventional fixed appliances. ${ }^{2}$ Orthodontic treatment with clear aligners is a quickly growing sector in the field of Orthodontics. ${ }^{3}$ Experienced clinicians have found that the clear aligner therapy for complex malocclusion often fails to yield the same degree of precision anticipated with fixed appliances. This necessitates careful case selection and counseling when offering this treatment to patients. A suitable case has been selected, illustrating the ease and simplicity with which the clear aligners can be used for treating certain malocclusions where the patient was reluctant to undergo fixed orthodontic therapy. ${ }^{4}$

\section{Case Report}

A 53 year old female patient reported with a chief complaint of spacing and forwardly placed upper front teeth. She had class I molar relation, proclined maxillary and mandibular anteriors, generalized spacing of $7 \mathrm{~mm}$ in maxillary and $3 \mathrm{~mm}$ in mandibular arch, overjet of $8 \mathrm{~mm}$ and overbite of $4 \mathrm{~mm}$, overall good periodontal support, Bolton's discrepancy showed a $2.5 \mathrm{~mm}$ tooth material excess in the lower arch, ANB of $2^{0}$ and incompetent lips (Table 2, Fig. 1-3). It was decided to treat the patient with clear aligners as only tipping of the anterior teeth was needed without complex tooth movement and to address the esthetic concerns of the patient. The available space in the upper and lower arch was used to retract the proclined teeth. The computer-generated virtual setup developed by the aligner company was reviewed, modified, and accepted (Fig. 4 $\mathrm{a} \& \mathrm{~b})$. The case was treated with 15 aligners in the upper arch and 10 aligners in the lower arch with no interproximal reduction indicated until the later. Stages of treatment to correct the Bolton's discrepancy which indicated a tooth material excess in the lower arch.

\section{Treatment Progress}

Zero aligners are passive aligners with no tooth movements incorporated were delivered within 48 hours of the PVS impressions sent to the laboratory so that the patient gets used to wearing the aligners. First, set of upper and lower aligners (U1, L1) were delivered on the 5th day and the patient asked to wear it for nearly 22 hours for 3 weeks a day except while eating. The patient was seen every 3 to 4 weeks to check for the aligner fit, any breakage, treatment progress, and patient cooperation in regularly wearing the aligners. The overall treatment duration was 1 year 1 month. Canine to canine upper and lower bonded fixed retainers were placed to maintain the corrections achieved. The following table below shows the duration of each aligner worn by the patient. (Table 1)

Table 1: The upper and lower aligners were changed every 3-4weeks accordingly as and when the current aligners showed a loose fit. The lower $10^{\text {th }}$ aligner was continued wearing till the end of the treatment until upper $15^{\text {th }}$ aligner which was the last aligner.

\begin{tabular}{|c|c|c|}
\hline Upper Aligner & Lower Aligner & Duration \\
\hline U1 & L1 & 3 Weeks \\
\hline U2 & L2 & 4 Weeks \\
\hline U3 & L3 & 3 Weeks \\
\hline U4 & L4 & 4 Weeks \\
\hline U5 & L5 & 4 Weeks \\
\hline U6 & L6 & 4 Weeks \\
\hline U7 & L7 & 4 Weeks \\
\hline U8 & L8 & 3 Weeks \\
\hline U9 & L9 & 3 Weeks \\
\hline U10 & L10 & 4 Weeks \\
\hline U11 & L10 & 4 Weeks \\
\hline U12 & L10 & 4 Weeks \\
\hline U13 & L10 & 3 Weeks \\
\hline U14 & L10 & 4 Weeks \\
\hline U15 & L10 & 3 Weeks \\
\hline \multicolumn{2}{|c}{}
\end{tabular}




\section{Treatment Results}

The final result showed a fairly good alignment in the upper and lower arches with a mild degree of open bite in the posteriors which did settle down after 6 months of treatment completion due to occlusal biting forces. (Fig. 9)

At the end of the treatment all the spaces were closed and the maxillary and mandibular incisors were kept upright, the molar and canine were in classic relationship, lip strain reduced and lip Competency achieved and an ideal overbite and overjet was achieved, as compared favorably with many published cases treated with fixed appliances. (Table 2) (Fig. 6-8)

Mesiobuccal rotation of 31 was left unresolved as the patient was satisfied with the treatment outcome and did not want any further refinement aligners to resolve the rotated 31.

Table 2: Pretreatment and postreatment cephalometric parameters

\begin{tabular}{|l|c|c|}
\hline Cephalometric Parameters & Pre-Treatment Values & Post-Treatment Values \\
\hline SNA & $82^{\circ}$ & $80^{\circ}$ \\
\hline SNB & $80^{\circ}$ & $80^{\circ}$ \\
\hline ANB & $2^{\circ}$ & $2^{\circ}$ \\
\hline Upper incisor to NA $(<) / \mathrm{mm}$ & $34^{0} / 8 \mathrm{~mm}$ & $28^{0} / 5 \mathrm{~mm}$ \\
\hline Lower incisor to NB $(<) / \mathrm{mm}$ & $38^{0} / 7 \mathrm{~mm}$ & $32^{\circ} / 4 \mathrm{~mm}$ \\
\hline Nasolabial Angle & $122^{0}$ & $100^{\circ}$ \\
\hline Lip Strain & $2 \mathrm{~mm}$ & $0 \mathrm{~mm}$ \\
\hline Interincisal Angle & $107^{0}$ & $140^{\circ}$ \\
\hline
\end{tabular}
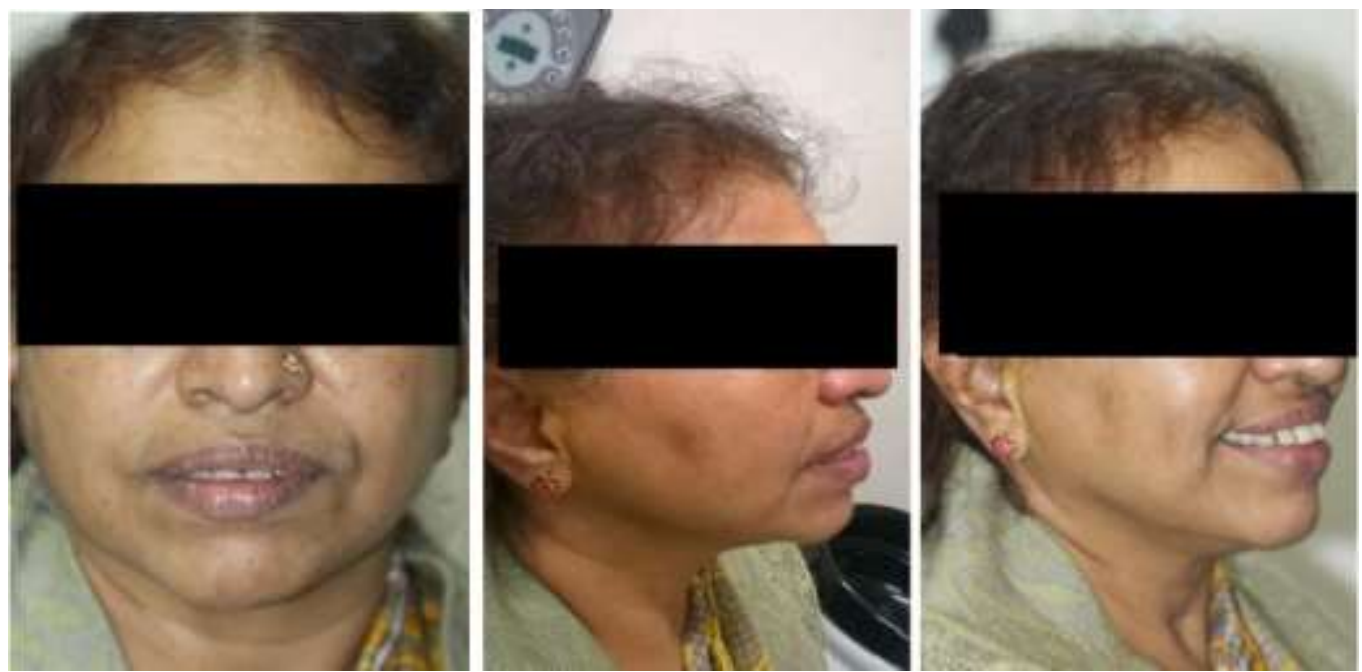

Fig. 1: Pre-treatment extraoral photos
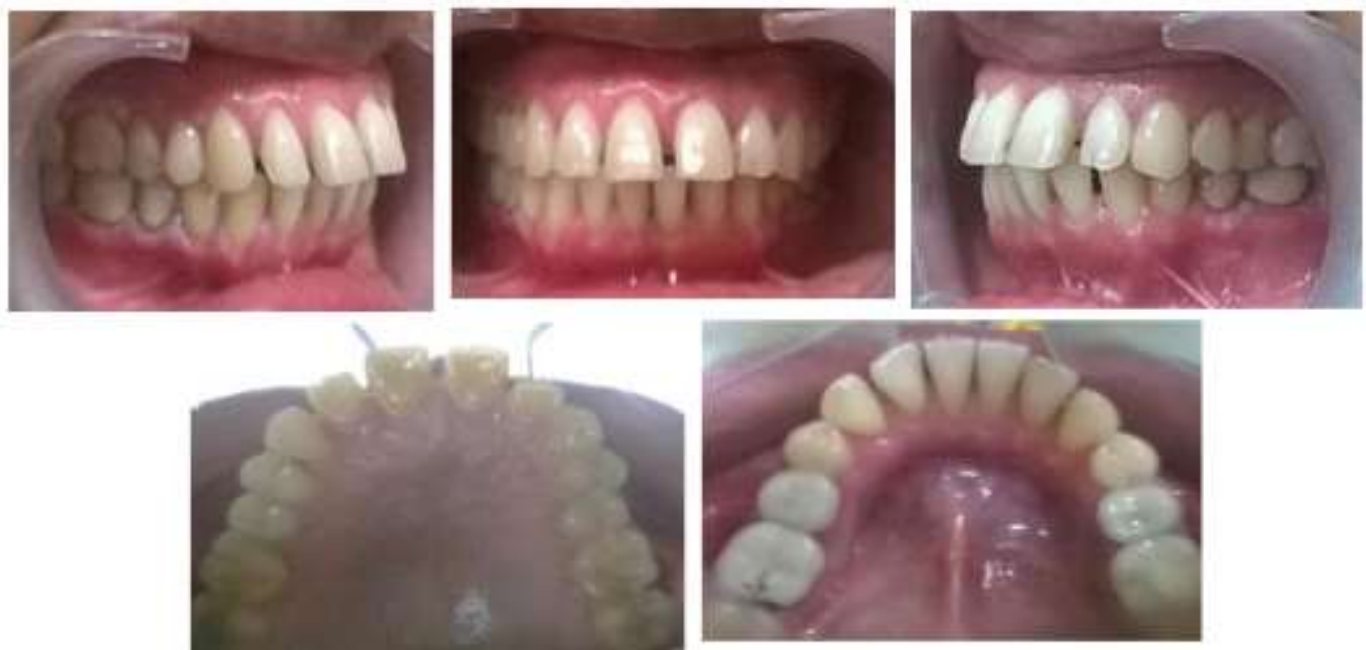

Fig. 2: Pre-treatment intraoral photographs 


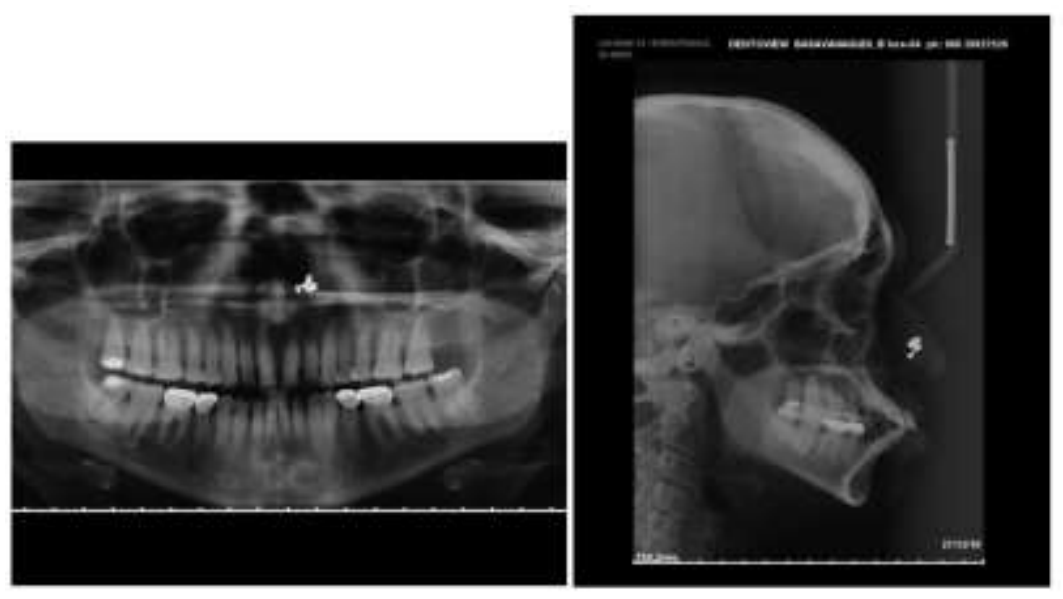

Fig. 3: Pre-treatment OPG and lateral cephalogram
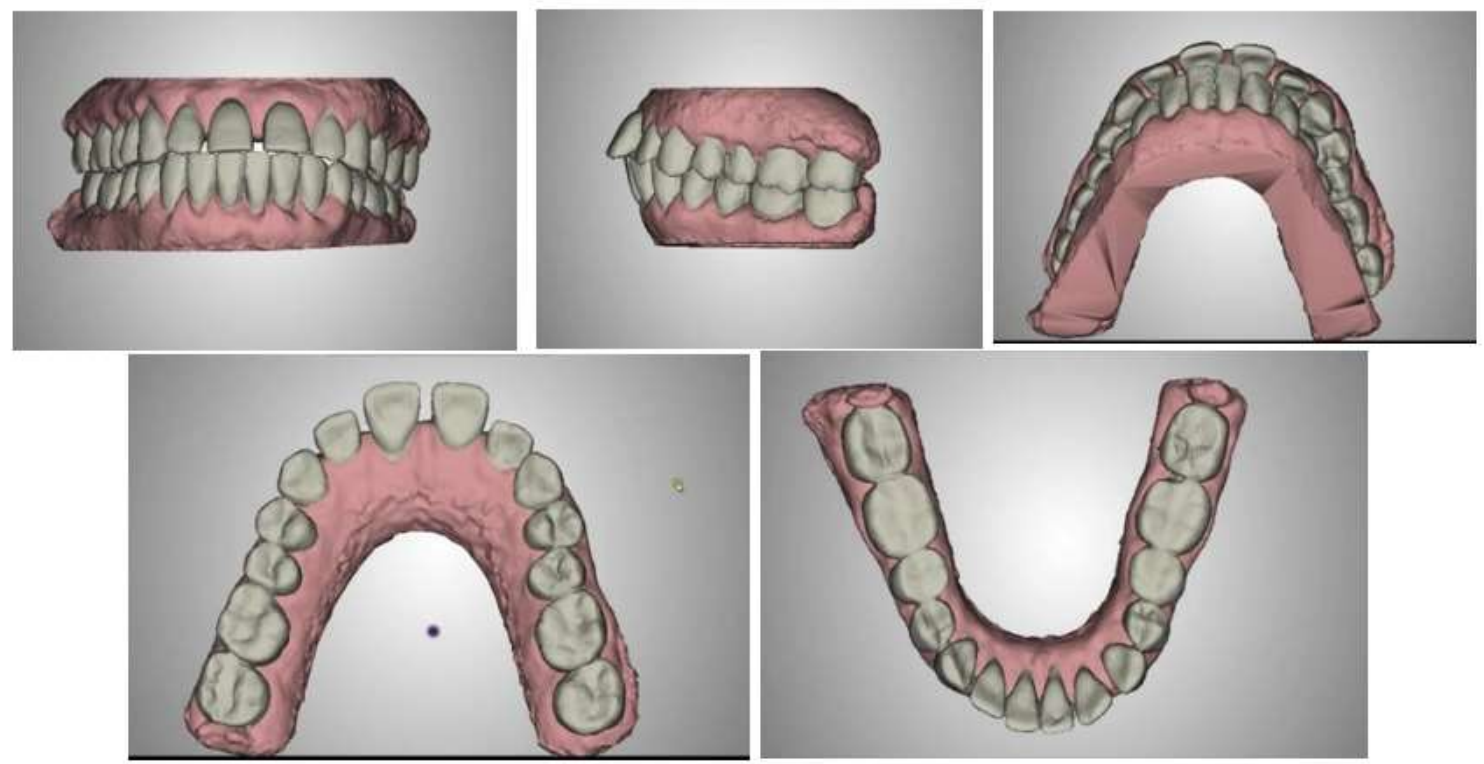

Fig 4a: Pre-treatment virtual set up model of the patient
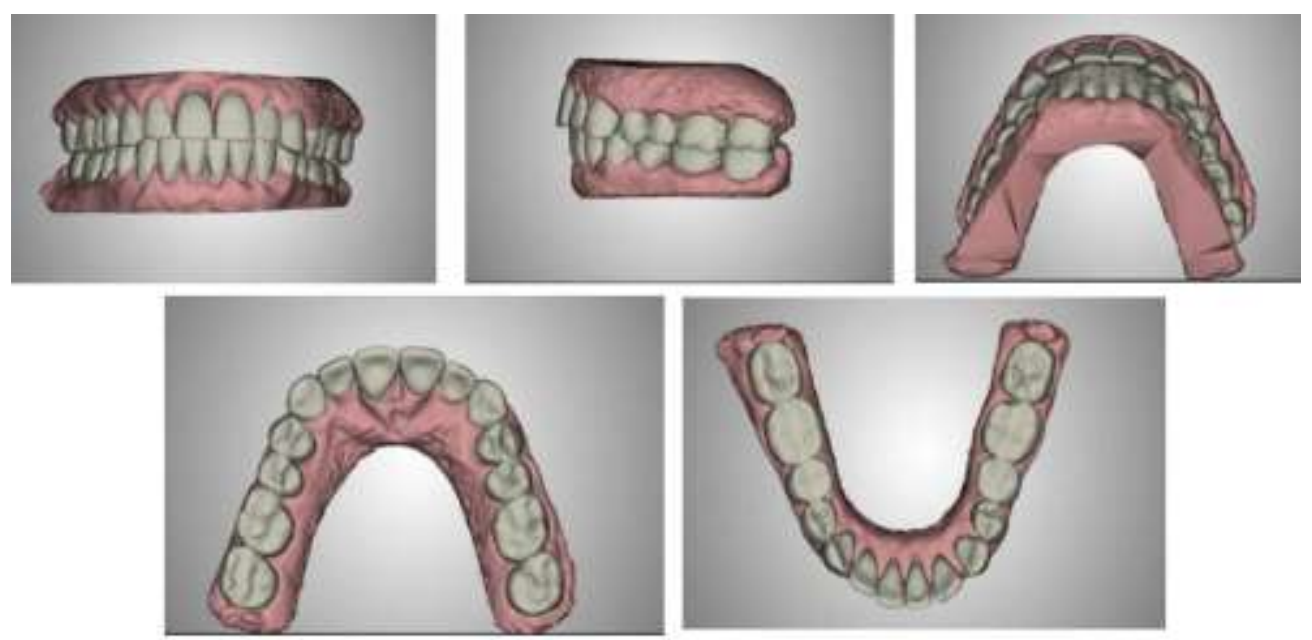

Fig. 4b: Post-treatment virtual set up model of the patient 


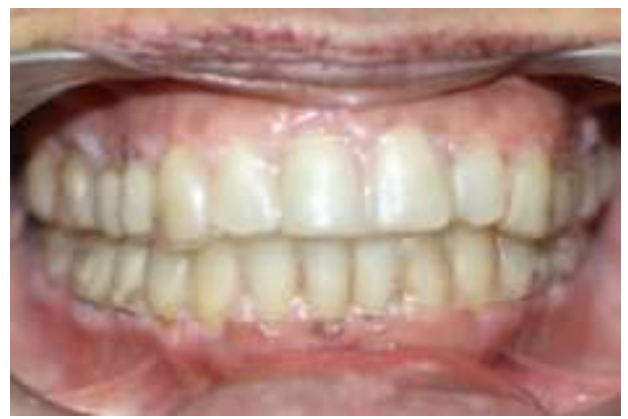

Fig. 5: Clear aligners worn in the upper and lower arch during mid-treatment post space closure
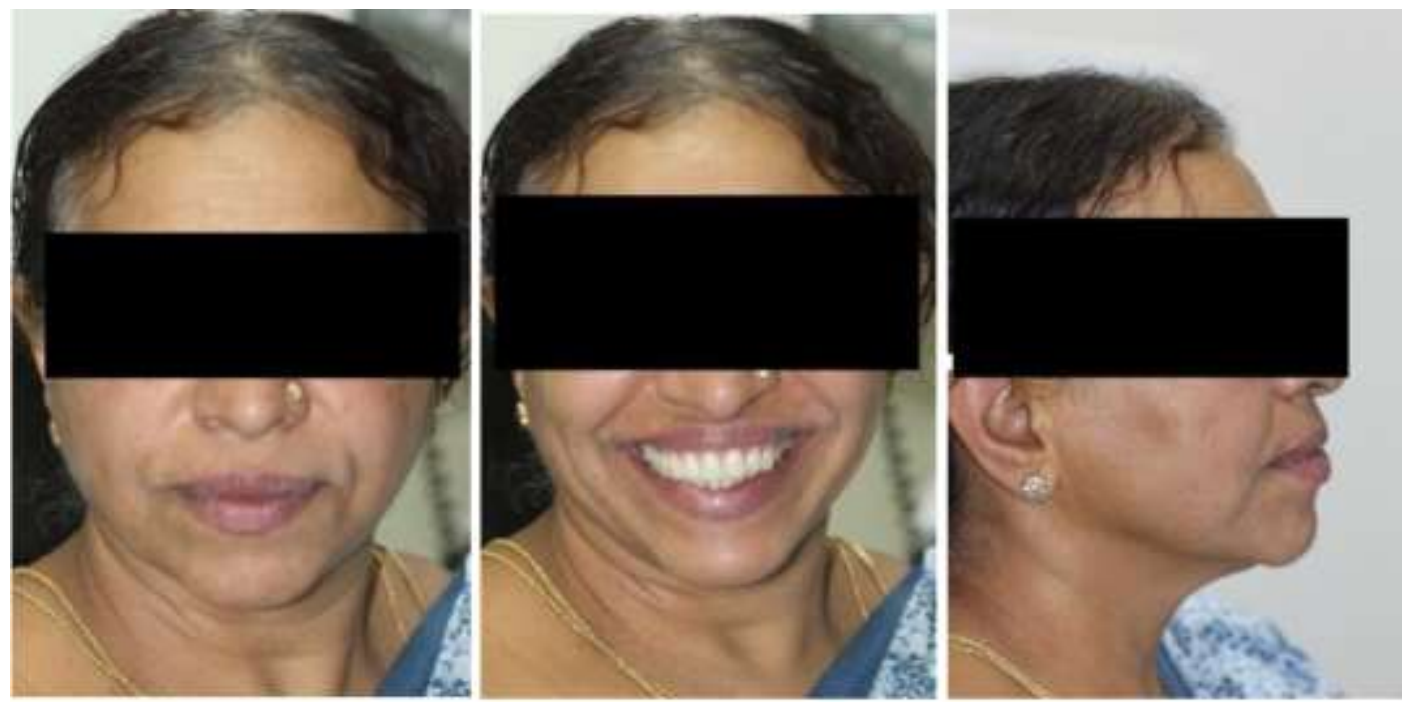

Fig. 6: Post-treatment extraoral photographs
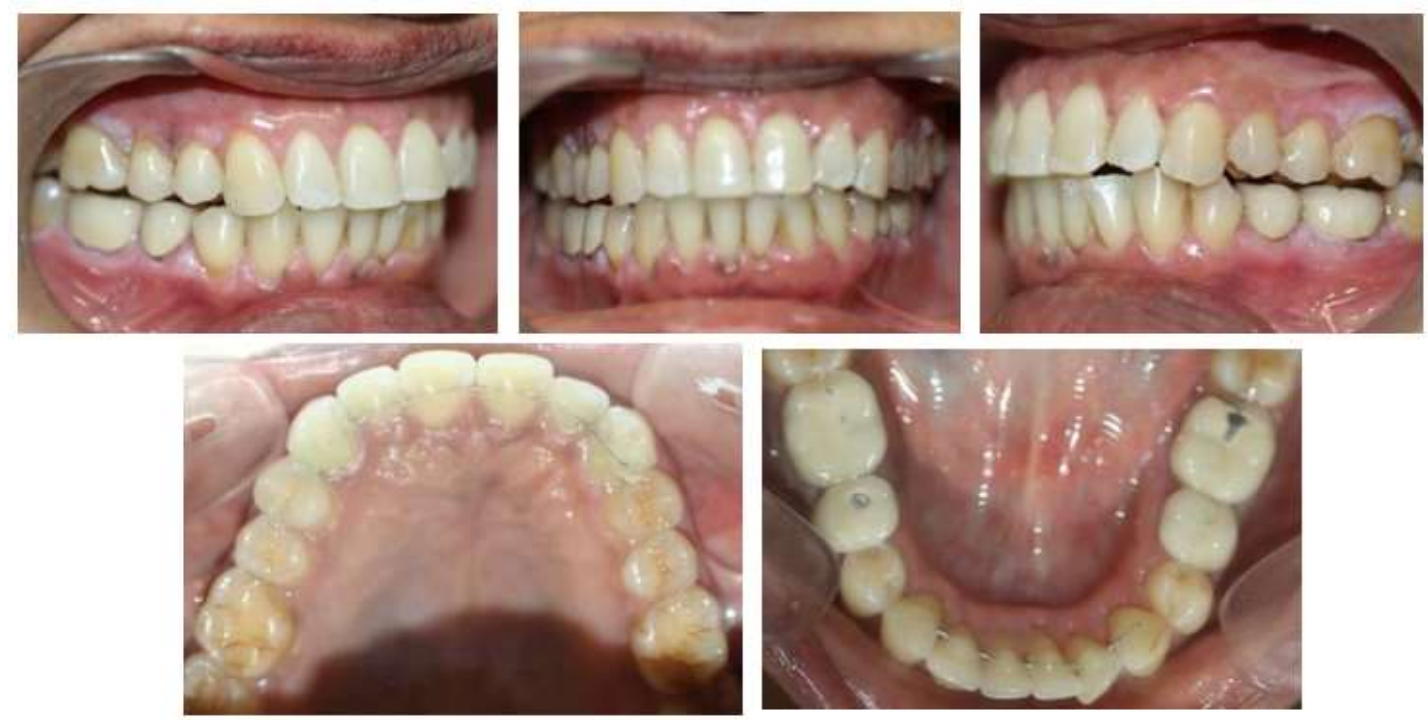

Fig. 7: Post-treatment intraoral photographs 

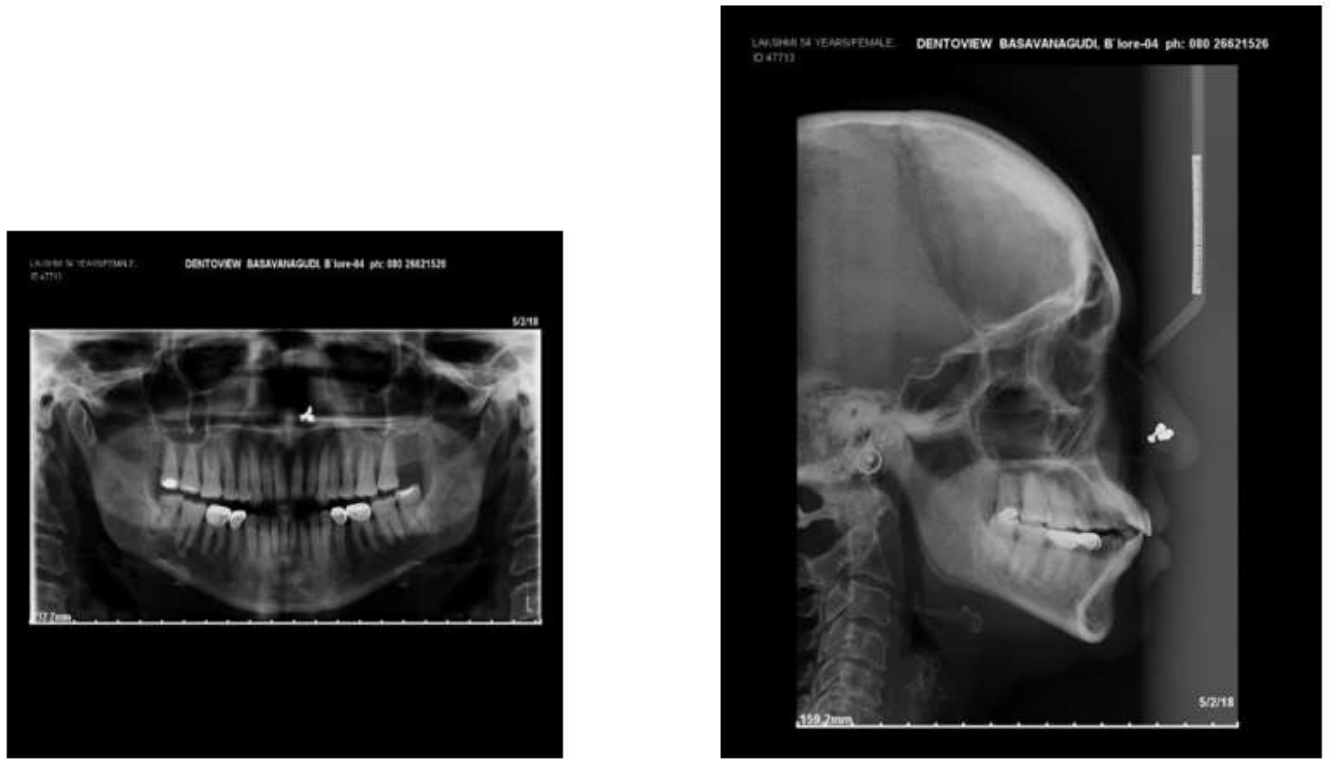

Fig 8: Post-treatment OPG and lateral cephalogram
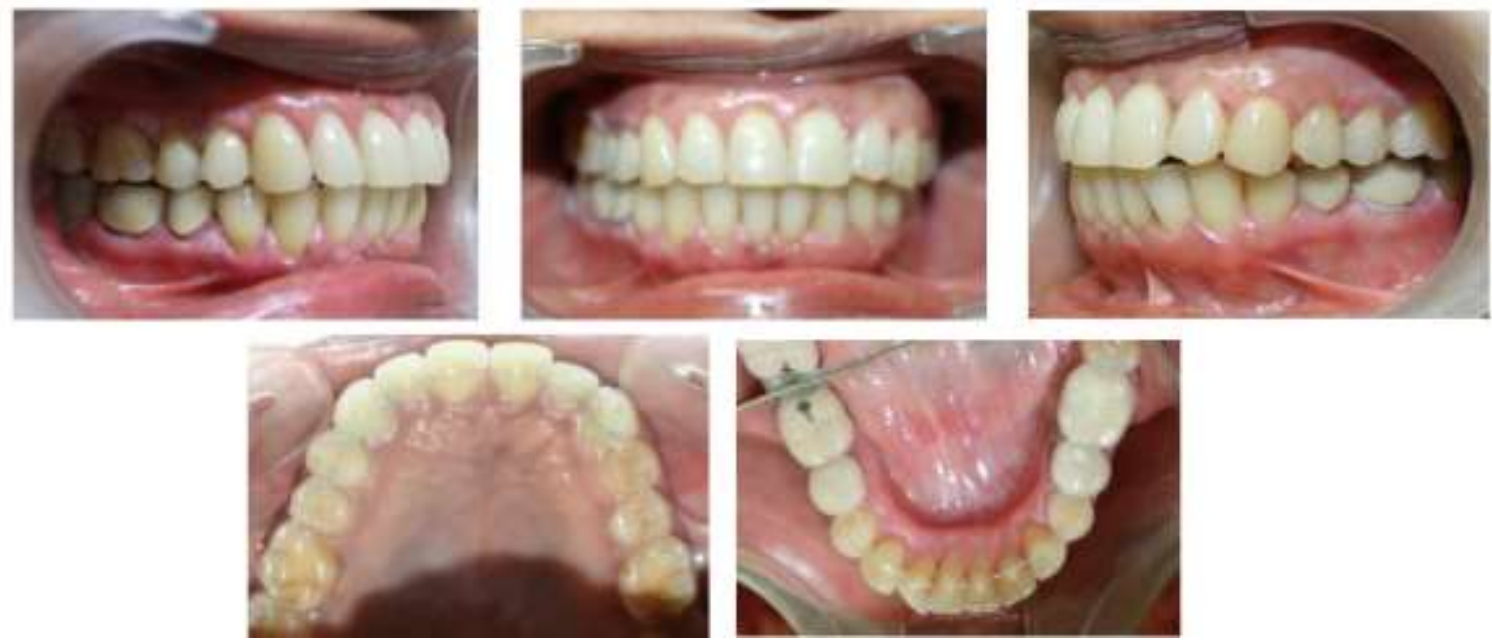

Fig. 9: Post -treatment intraoral photographs taken 6 months after treatment showing posterior occlusal settlement

\section{Discussion}

Clear Aligners have become the current generation treatment option for adults wanting to undego orthodontic tooth correction with esthetic concerns. However, patient selection for clear aligner therapy is very crucial and predictable results can be achieved in mild to moderate tooth corrections involving space closure, retraction and decrowding.

It is important to compare the clinical positions of the teeth with the virtual setup images after every 4-5 sets of aligners. If the comparison is not within $10 \%$, the clinician should stop the treatment at that point and ask the laboratory to deliver a new set of aligners with all the necessary corrections incorporated into the aligners.

In this case report, the efficacy of the clear aligner can be appriciated where the proclination, spacing of the anterior teeth has been treated. The patient reported with a good periodontal status which was maintained during the treatment and post treatment as well. The overjet of $8 \mathrm{~mm}$ was reduced to $2 \mathrm{~mm}$ and the spacing of $7 \mathrm{~mm}$ in the upper arch and $3 \mathrm{~mm}$ in the lower arch were closed. Mesiobuccal rotation of 31 was left unresolved as the patient was satisfied with the treatment outcome and did not want any further refinement aligners to resolve the rotated 31 . There was an overall improvement in the facial profile with decreased lip strain, increased nasolabial angle and lip competancy was achieved.

\section{Conclusion}

Clear Aligners are an effective and esthetic mode of orthodontic tooth movement in adults provided the case has been accordingly selected as complex tooth movements and very precise occlusal finishing is difficult to achieve with aligners. Simple tooth movements like proclination and spacing can be corrected along with an overall improvement in the facial esthetics as seen in the above mentioned case report. 


\section{Source of Funding}

None.

\section{Conflict of Interest}

None.

\section{References}

1. Kesling HD. Coordinating the predetermined pattern and tooth positioner with conventional treatment. Am J Orthod Oral Surg. 1946;32:285-93.

2. Rossinia G, Parrini S. Efficacy of clear aligners in controlling orthodontic tooth movement: A systematic review. Angle Orthod. 2015;85(5):881-9.

3. Orthodontic clear aligner treatment, Seminar in orthodontics. Semin Orthod. 2017;23(1):83-9.

4. Singh I, Aziz SB, Singh G. Predictable results using clear aligners: Case report. Srilankan J Orthod. 2018;1(1):1-11.

5. Womack WR. Four- Premolar extraction treatment with Invisalign. J Clin Orthod. 2006;XL(8):493-500.

6. Barlattani A, Mampieri G, Ottria L, Bollero P. Invisalign treatment in periodontal patient: case report. J Oral Implantol. 2009;2(4):35-9.

How to cite: Bharathi VS, Dharmesh HS, Kiran H. Clear aligner therapy for adult patient - A case report. Indian $J$ Orthod Dentofacial Res.2020;6(1):31-6. 